\title{
The octopus as paradigm for soft robotics
}

\author{
Matteo Cianchetti \\ The BioRobotics Institute, Scuola Superiore Sant'Anna, Pisa, Italy \\ (Tel : +39-050-883406; E-mail: matteo.cianchetti@sssup.it)
}

\begin{abstract}
Looking at an octopus from the roboticist view point it is easy to understand why it is considered a paradigmatic example for soft robotics: its arms are soft and deformable, they can bend in any direction, at any point along the arm; however, they can stiffen when needed and they can grasp and pull objects with considerable strength; the octopus does not have a large brain, yet it can control this huge amount of possible movements and motion parameters. These observations together with the growing need for robots in service tasks, in unstructured environments, in contact with humans, is leading to release the basic assumption of rigid parts in robotics and to the development of new enabling technologies for a new generation of soft robots for marine and surgical robotics.
\end{abstract}

Keywords - Bioinspiration, biomimetics, soft robotics.

\section{Introduction}

The 50-year robotics history is based on the assumption that robots are kinematic chains of rigid links, on which robot control theories and techniques have developed so far, providing extreme accuracy in robot operations. On the other hand, there is a growing need for robots in service tasks, in unstructured environments, in contact with human beings. By taking inspiration from the variety of organisms inhabiting our natural environment, the need for soft body parts appears clear, to increase adaptability and robustness. In addition to this, modern views of artificial intelligence attribute a stronger role to the physical body and to the interaction with the environment, in so-called embodied intelligence, or morphological computation. Again, compliance, or softness, are needed for implementing these principles, for developing bodies that can react to the environment, compensate external forces, and ultimately simplify control.

Soft robotics is not just a new direction of technological development. The use of soft materials in robotics is going to unhinge its fundamentals. Soft robotics is going to stand as a novel approach to robotics and artificial intelligence, and it has the potential to produce a new generation of robots, in the support of humans in our natural environments. The octopus is undoubtedly a good model for soft robotics, and an extreme one, considering that it has no rigid structures, of any kind.

\section{New science and technology}

Roboticists who study biological systems look at the knowledge of the biological principles and their generalization, to take inspiration for the design of novel products. Bioengineering and biological methods are applied to study, measure and model octopus performance, with results of new scientific data beyond the state of the art, as well as novel design principles and specifications for robotics purpose. This abstraction pushed the development of new technologies for soft robot bodyware, soft actuators, soft tactile and proprioceptive systems, a stretchable skin, and suckers.

Modelling and controlling such soft robots represent a new set of fundamental problems. At the same time, soft bodies amplify the possibility to put in place morphological computation mechanisms, which simplify control and provide the opportunity for exploring and developing novel techniques.

Different prototypes of soft arms have been developed, basically composed of silicone, driven by cables and shape memory alloys (SMA) technologies. An "artificial muscular-hydrostat" unit has been developed to replicate the same octopus movements and it is used as fundamental unit in an octopus-like soft robotic arm for movement and manipulation [1]. Arm models and control architecture have been developed to move the arm as the real octopus. The arm is used in water and it is able to elongate, shorten, and pull, as well as to bend in all the directions (Fig. 1). Furthermore, it is capable to grasp objects, as a bottle or a human hand, by adapting its shape to the target. A sensitive skin, with contact sensors embedded into silicone rubber, surrounds the arm with passive suckers allowing the grasping of objects.

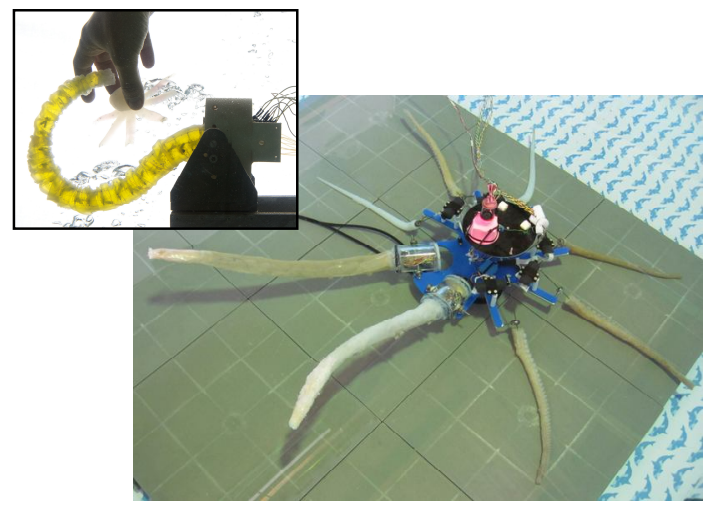

Fig. 1. The OCTOPUS arm (up-right) and the complete octopus-like robotic platform.

With no rigid structures and eight flexible arms (Fig. 1) OCTOPUS is able to reach impracticable places and simultaneously showing manipulation capability, which could open up new scenarios for marine exploration and 
underwater rescue.

The octopus-like robot is a good example of the feasibility of soft robots and related technologies, but it is also taking its way towards helpful applications: a 'grown-up' octopus robot helping humans in underwater explorations (section 3) and operations and a soft manipulator for biomedical applications (section 4).

\section{New paradigm for marine robotics}

Starting from the knowledge and the technologies derived from the study of the octopus a straightforward application filed is represented by the marine robotics where the motion strategies and capabilities can take advantage of its most adapt environment. The use of soft robots in underwater applications represents a very innovative application, perfectly complementary to the current use of underwater robot, as a soft robot can get in contact with the environment, crawl on the sea bottom and reefs, stick on plants or get inside structures to explore.

Exploration tasks for archaeology, petroleum engineering, harbour and off-shore engineering are a few out of many application scenarios where such a robot could demonstrate the effectiveness of soft robotics based solution, and show the advantages over the state of the art, in terms of manipulation, swimming and locomotion capabilities obtained.

This is the perfect synthesis of the envisaged capabilities of the PoseiDRONE robot [2], an innovative soft robot able to swim, locomote on different substrates and to manipulate objects in narrow spaces. Moreover the added value of such a system, compared to the currently known underwater robots, is represented by the combination of advanced functionalities conjugated with high safety (for the robot and the environment), extreme adaptability and low cost.

\section{New frontiers in surgery}

The octopus has been an inspiration source also in the biomedical field where traditional robotic tools find limitations in terms of dexterity and softness. In minimally invasive surgery, for example, traditional laparoscopic operations need several trocar accesses because of the limited dexterity, flexibility, manoeuvrability of the available tools. Based on these limitations a different approach has been pursued for the development of the STIFF-FLOP manipulator [3], taking inspiration from the manipulation capabilities of the octopus arm. Despite with different technological solutions then the OCTOPUS arm, the manipulator is again designed to present elongation, squeezing and large flexibility in bending, but also contemporary showing stiffness changing capabilities.

The basic ideas stolen from the octopus have been applied in a soft manipulator able to turn from a completely soft state into a state of precise and, if needed, powerful articulation.

The manipulator is based on a series of identical modules, each one consisting of a silicone tube with flexible fluidic actuators for enabling omnidirectional bending and elongation capability (Fig. 3) and one central channel for exploiting the granular jamming phenomenon for a selective stiffness changing (Fig. 3).

It is worth mentioning that when in passive state, the module can be highly deformed and squeezed without affecting its functionalities (Fig. 3). This feature could be also employed to avoid the risk of damaging both the manipulator and the organs and to fit with different trocar diameters.
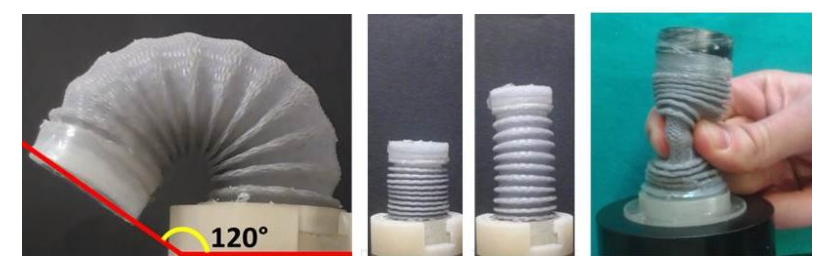

Fig. 3. STIFF-FLOP module capabilities in terms of bending, elongation and squeezability.

\section{Conclusions}

The role of soft body parts appears clear in natural organisms, to increase adaptability and robustness. Compliance, or softness, are also needed for implementing the principles of embodied intelligence, or morphological computation, a modern view of intelligence, attributing a stronger role to the physical body and its interaction with the environment. The combined application of these principles can lead to the development of new kinds of robots which can be applied in several application fields. Exploiting the advantages associated with both soft and hard systems this new generation of robots can achieve high dexterity, adaptability, resilience in environment overcoming the difficulties encountered by traditional robots based on rigid structures.

\section{Acknowledgement}

This works have been supported by the European Commission with the OCTOPUS IP (\#231608, FET Proactive Initiative ICT 2007.8.5 "Embodied Intelligence") and the STIFF-FLOP IP (\#287728, ICT Challenge 2) and by the Fondazione Cassa di Risparmi di Livorno with the PoseiDRONE e PoseiDRONE II projects.

The author wishes to acknowledge the work of the project partners and of the SSSA Soft Robotics Team: Cecilia Laschi, Arianna Menciassi, Laura Margheri, Andrea Arienti, Marcello Calisti, Francesco Giorgio Serchi, Maurizio Follador.

\section{References}

[1] C. Laschi, B. Mazzolai, M. Cianchetti, L. Margheri, M. Follador, P. Dario "A Soft Robot Arm Inspired by the Octopus", Advanced Robotics (Special Issue on Soft Robotics), 26 (7) 709-727, 2012.

[2] http://sssa.bioroboticsinstitute.it/projects/PoseiDRONE.

[3] M. Cianchetti, T. Ranzani, G. Gerboni, I. De Falco, C. Laschi, A. Menciassi, "STIFF-FLOP Surgical Manipulator: mechanical design and experimental characterization of the single module" to appear in Proceedings of IEEE on Intelligent and Robotic Systems - IROS 2013. 